\title{
Descent systems for Bruhat posets
}

\author{
Lex E. Renner
}

Received: 9 May 2007 / Accepted: 9 May 2008 / Published online: 29 May 2008

(C) Springer Science+Business Media, LLC 2008

Abstract Let $(W, S)$ be a finite Weyl group and let $w \in W$. It is widely appreciated that the descent set

$$
D(w)=\{s \in S \mid l(w s)<l(w)\}
$$

determines a very large and important chapter in the study of Coxeter groups. In this paper we generalize some of those results to the situation of the Bruhat poset $W^{J}$ where $J \subseteq S$. Our main results here include the identification of a certain subset $S^{J} \subseteq W^{J}$ that convincingly plays the role of $S \subseteq W$, at least from the point of view of descent sets and related geometry. The point here is to use this resulting descent system $\left(W^{J}, S^{J}\right)$ to explicitly encode some of the geometry and combinatorics that is intrinsic to the poset $W^{J}$. In particular, we arrive at the notion of an augmented poset, and we identify the combinatorially smooth subsets $J \subseteq S$ that have special geometric significance in terms of a certain corresponding torus embedding $X(J)$. The theory of $\mathcal{g}$-irreducible monoids provides an essential tool in arriving at our main results.

Keywords Weyl group · Bruhat poset - Descent system - Augmented poset · g-irreducible monoid $\cdot$ Rationally smooth

\section{Introduction}

If ( $W, S$ ) is a Weyl group and $w \in W, s \in S$, then either $w s<w$ or else $w<w s$. Hence we define

$$
D(w)=\{s \in S \mid l(w s)<l(w)\}
$$

L.E. Renner $(\bowtie)$

Department of Mathematics, University of Western Ontario, London, N6A 5B7, Canada

e-mail: lex@uwo.ca 
the descent set of $w \in W$. This innocuous looking situation is at the heart of many important results in geometry, combinatorics, group theory and representation theory.

Evidently, the interest in these objects began with Solomon [17], who defines a certain subalgebra $B \subseteq \mathbb{Q}[W]$, and uses it to help understand the representations of $W$. The algebra $B$ is often called the descent algebra since it can be defined in terms of descent sets. Brown [5] looks at this descent algebra and reconstitutes it as the semigroup algebra of a certain idempotent ("face") semigroup associated with the reflection arrangement of $W$.

The numbers $|D(w)|$ can be used to calculate the Betti numbers of the associated torus embedding $X(\emptyset)$ of $W$. These Betti numbers can be obtained directly from the $h$-vector of the associated rational, convex polytope. In [19] Stanley proves that the $h$-vector of any simplicial, convex polytope is a symmetric, unimodal sequence. Stembridge [16] proves that the canonical representation of $W$ on $H^{*}(X(\emptyset) ; \mathbb{Q})$ is a permutation representation and, with the help of Dolgachev-Lunts [8], he computes this representation. In [3] Brenti studies these descent polynomials (i.e. the Poincaré polynomials of $X(\emptyset)$ ) as analogues of the Eulerian polynomials. He also looks at the $q$-analogues of these polynomials.

In the theory of group embeddings $|D(w)|$ is an important ingredient in the calculation of the Betti numbers of the "wonderful" compactification of a semisimple group of adjoint type. See [7, 14].

In this paper we expand the entire study to include all Bruhat posets $W^{J}$, where $J \subseteq S$. In particular, we study the relationship between $W^{J}$ and a certain torus embedding $X(J)$. This leads us to the notion of an augmented poset $\left(W^{J}, \leq\right.$, $\left.\left\{v_{s}\right\}_{s \in S \backslash J}\right)$. This ordering on $W^{J}$ is not the usual Bruhat order on $W^{J}$. It is quantified in terms of a certain "ascent/descent" structure on $W^{J}$. Further analysis leads us to the notion of a descent system $\left(W^{J}, S^{J}\right)$. These descent systems are particularly interesting if $X(J)$ is quasi-smooth in the sense of Danilov [6]. In the remainder of this paper we refer to this condition as rationally smooth, which is currently the accepted terminology. For the convenience of the reader we give a precise definition.

Definition 0.1 Let $X$ be a complex algebraic variety of dimension $n$. Then $X$ is $r a$ tionally smooth at $x \in X$ if there is a neighbourhood $U$ of $x$ in the complex topology such that, for any $y \in U$,

$$
H^{m}(X, X \backslash\{y\})=(0)
$$

for $m \neq 2 n$ and

$$
H^{2 n}(X, X \backslash\{y\})=\mathbb{Q} .
$$

Here $H^{*}(X)$ denotes the cohomology of $X$ with rational coefficients.

See [4] for a modern account of this key notion, along with some important results about rationally smooth varieties with torus action.

The main point of this paper is to identify and study the set

$$
\{J \subseteq S \mid X(J) \text { is rationally smooth }\}
$$


See Theorem 3.2 and Corollary 3.5 below. The descent system $\left(W^{J}, S^{J}\right)$ leads to a useful combinatorial analogue of the method of Bialynicki-Birula [1]. This allows us to uncover some of the finer geometry of $X(J)$. The main results of this paper could be stated entirely in the language of Weyl groups, root systems and polytopes. However, we were led to these results by trying to calculate the Betti numbers of a certain class of projective varieties that arise naturally from the theory of reductive monoids. It turns out that "step one" of this monoid problem required that we quantify the Betti numbers of $X(J)$. This eventually requires that we quantify the "ascent/descent" structure on $W^{J}$ for certain $J$. Reductive monoids are an essential tool in showing us how to do this.

\section{$1 W$-invariant polytopes}

Let $V$ be a rational vector space and let $r: W \rightarrow G L(V)$ be the usual reflection representation of the Weyl group $W$. Along with this goes the Weyl chamber $\mathcal{C} \subseteq V$ and the corresponding set of simple reflections $S \subseteq W$. The Weyl group $W$ is generated by $S$, and $\mathcal{C}$ is a fundamental domain for the action of $W$ on $V$. See Chapter III of [10] for a detailed discussion of Weyl groups.

Let $\lambda \in \mathcal{C}$. In this section we describe the face lattice $\mathcal{F}_{\lambda}$ of the polytope

$$
\mathcal{P}_{\lambda}=\operatorname{Conv}(W \cdot \lambda)
$$

the convex hull of $W \cdot \lambda$ in $V$. The face lattice $\mathcal{F}_{\lambda}$ depends only on $W_{\lambda}=\{w \in$ $W \mid w(\lambda)=\lambda\}=W_{J}=\langle s \mid s \in J\rangle$, where $J=\{s \in S \mid s(\lambda)=\lambda\}$. Thus we describe $\mathcal{F}_{\lambda}=\mathcal{F}_{J}$ explicitly in terms of $J \subseteq S$.

Closely associated with these polytopes is a certain class of reductive, algebraic monoids. We use what is known about this class of monoids to calculate $\mathcal{F}_{J}$ in terms of the underlying Dynkin diagram of $(W, S)$.

We now recall some results first recorded in [12]. Throughout the paper we use the language and techniques of linear algebraic monoids. Unfortunately this theory is not widely appreciated, but luckily the main results and constructions have recently been assembled in [15]. See, especially, Chapters 4, 5, 7, and 8 of [15]. Throughout the paper we work over the field $\mathbb{C}$ of complex numbers. That is, all algebraic groups and monoids are assumed to be algebraic varieties defined over $\mathbb{C}$. Much of what is said in this paper is valid over any algebraically closed field. Restricting our discussion to varieties over the complex numbers is required only for the sake of Definition 0.1. Let $M$ be an irreducible, normal algebraic monoid with reductive unit group $G$. We refer to such monoids as reductive. The reader can find any unproved statements about reductive monoids in $[11,15]$. See Solomon's survey [18] for a less technical introduction to the subject.

If $M$ is a reductive monoid with unit group $G$ we let $B \subseteq G$ be a Borel subgroup of $G$ and $T \subseteq B$ a maximal torus of $G$. We let $\bar{T}$ denote the Zariski closure of $T$ in $M$. By part b) of Theorem 4.5 of [15], $\bar{T}$ is a normal, affine torus embedding. The set of idempotents $E(\bar{T})$ of $\bar{T}$ is defined to be

$$
E(\bar{T})=\left\{e \in \bar{T} \mid e^{2}=e\right\} .
$$


There is exactly one idempotent in each $T$-orbit on $\bar{T}$. In the cases of interest in this paper, $E(\bar{T}) \backslash\{0\}$ can be canonically identified (as a poset) with the face lattice $\widetilde{F}_{\lambda}$ for appropriate $\lambda \in \mathcal{C}$. It turns out that this poset structure on $E(\bar{T})$ is given by

$$
e \leq f \text { if } e f=e \text {. }
$$

We note that $e \leq f$ if and only if $e T \subseteq \overline{f T}$. We let $E_{1}=E_{1}(\bar{T})=\{e \in E(\bar{T}) \mid$ $\operatorname{dim}(T e)=1\}$. In the above-mentioned identification, $E_{1}$ is identified with the vertices of $\mathcal{F}_{\lambda}$. We shall see that the combinatorial structure of $E_{1}$ is much richer because $\bar{T}$ comes from the reductive monoid $M$.

The $G \times G$-orbits of $M$ are particularly important throughout this paper. Let

$$
\Lambda=\{e \in \bar{T} \mid e B=e B e\}
$$

be the cross section lattice of $M$ relative to $T$ and $B$. See Chapter 9 of [11]. It is a basic fact that

$$
M=\bigsqcup_{e \in \Lambda} G e G,
$$

where $G e G \subseteq \overline{G f G}$ if and only if $e f=e$. See Theorem 4.5 of [15].

As above we let $S \subseteq W$ be the set of simple involutions of $W$ relative to $T$ and $B$. We can regard $S$ as the set of vertices of a graph with edges $\{(s, t) \mid s t \neq t s\}$. Thus we may speak of the connected components of any subset of $S$.

A reductive monoid $M$ with $0 \in M$ is called $g$-irreducible if $M \backslash\{0\}$ has exactly one minimal $G \times G$-orbit. See [12], or Section 7.3 of [15] for a systematic discussion of this important class of reductive monoids, and for a proof of the following Theorem.

Theorem 1.1 Let $M$ be a reductive monoid. The following are equivalent.

1. $M$ is g-irreducible.

2. There is an irreducible rational representation $\rho: M \rightarrow E n d(V)$ which is finite as a morphism of algebraic varieties.

3. If $\bar{T} \subseteq M$ is the Zariski closure in $M$ of a maximal torus $T \subseteq G$ then the Weyl group $W$ of $T$ acts transitively on the set of minimal nonzero idempotents of $\bar{T}$.

Notice in particular that one can construct, up to finite morphism, all g-irreducible monoids from irreducible representations of a semisimple group. Indeed, let $G_{0}$ be semisimple and let $\rho: G_{0} \rightarrow E n d(V)$ be an irreducible representation. Define $M_{1} \subseteq \operatorname{End}(V)$ to be the Zariski closure of $\mathbb{C}^{*} \rho\left(G_{0}\right)$ where $\mathbb{C}^{*} \subseteq \operatorname{End}(V)$ is the set of homotheties. Finally let $M(\rho)$ be the normalization of $M_{1}$. Then, according to Theorem 1.1, $M(\rho)$ is $g$-irreducible.

By the results of Section 4 of [12], if $M$ is $g$-irreducible, there is a unique, minimal, nonzero idempotent $e_{1} \in E(\bar{T})$ such that $e_{1} B=e_{1} B e_{1}$, where $B$ is the given Borel subgroup containing $T$. If $M$ is $g$-irreducible we say that $M$ is $g$-irreducible of type $J$ if, for this idempotent $e_{1}$,

$$
J=\left\{s \in S \mid s e_{1}=e_{1} s\right\},
$$


where $S$ is the set of simple involutions relative to $T$ and $B$. The set $J$ can be determined in terms of any irreducible representation satisfying condition 2 of Theorem 1.1. Indeed, let $\lambda \in X(T)_{+}$be any highest weight such that $\{s \in S \mid s(\lambda)=\lambda\}=$ $J$. Then $M\left(\rho_{\lambda}\right)$ is $g$-irreducible of type $J$ where $\rho_{\lambda}$ is the irreducible representation of $G_{0}$ with highest weight $\lambda$. The representation $\rho_{\lambda}$ determines a representation of $M\left(\rho_{\lambda}\right)$ on $V$. Furthermore, any two $g$-irreducible monoids with a finite, dominant morphism between them are of the same type. If $e_{1}$ is the above-mentioned minimal idempotent then $B^{-} e_{1}=e_{1} B^{-} e_{1}$, where $B^{-}$is the Borel subgroup opposite $B$. We observe that $e_{1} M e_{1}$ is a reductive monoid with idempotent set $\left\{0, e_{1}\right\}$ and thus $\operatorname{dim}\left(e_{1} M e_{1}\right)=1$. Hence $e_{1} B^{-} e_{1}$ is also one-dimensional. Thus there exists a character $\chi: B^{-} \rightarrow \mathbb{C}^{*}$ such that $b e_{1}=e_{1} b e_{1}=\chi(b) e_{1}$ for all $b \in B^{-}$. It follows that $B^{-}$acts on $e_{1}(V)$ by the rule

$$
\rho_{\lambda}(b)(v)=\rho_{\lambda}(b)\left(\rho_{\lambda}\left(e_{1}\right)(v)\right)=\chi(b) \rho_{\lambda}\left(e_{1}\right)(v)=\chi(b) v .
$$

Therefore $L=e(V) \subseteq V$ is the unique one-dimensional $\rho_{\lambda}\left(B^{-}\right)$-stable subspace of $V$ with weight $\lambda$. In particular, $\chi \mid T=\lambda$ and $P=\left\{g \in G_{0} \mid \rho_{\lambda}(g)(L)=L\right\}$ is a parabolic subgroup of $G_{0}$ of type $J$.

We now describe the $G \times G$-orbit structure of a $g$-irreducible monoid of type $J \subseteq S$. The following result was first recorded in [12].

\section{Theorem 1.2 Let $M$ be a g-irreducible monoid of type $J \subseteq S$.}

1. There is a canonical one-to-one order-preserving correspondence between the set of $G \times G$-orbits acting on $M$ and the set of $W$-orbits acting on the set of idempotents of $\bar{T}$. This set is canonically identified with $\Lambda=\{e \in E(\bar{T}) \mid e B=e B e\}$.

2. $\Lambda \backslash\{0\} \cong\{I \subseteq S \mid$ no connected component of $I$ is contained entirely in $J\}$ in such a way that e corresponds to $I \subseteq S$ if $I=\{s \in S \mid$ se $=e s \neq e\}$. If we let $\Lambda_{2}=\{e \in \Lambda \mid \operatorname{dim}(T e)=2\}$ then this bijection identifies $\Lambda_{2}$ with $S \backslash J$.

3. If $e \in \Lambda \backslash\{0\}$ corresponds to $I$, as in 2 above, then $C_{W}(e)=W_{K}$ where $K=$ $I \cup\{s \in J \mid s t=t s$ for all $t \in I\}$.

It is worthwhile to pause and notice that $\Lambda$ is completely determined by $J$. See [15] for a systematic discussion of g-irreducible monoids, in particular Lemma 7.8 of [15]. Notice also that part 1 of Theorem 1.2 is true for any reductive monoid. See Theorem 4.5 of [15] for more of those details.

Let $M$ be a $g$-irreducible monoid of type $J \subseteq S$ and assume that $\rho: M \rightarrow \operatorname{End}(V)$ is an irreducible representation which is finite as a morphism. Let $G$ be the unit group of $M$ with maximal torus $T \subset G$. Then let $G_{0}$ be the semisimple part of $G$ with maximal torus $T_{0}=G_{0} \cap T$, and let $\rho_{\lambda}=\rho \mid G_{0}$, with highest weight $\lambda \in \mathcal{C}$, the rational Weyl chamber of $G_{0}$. Then, as above, $J=\{s \in S \mid s(\lambda)=\lambda\}$. Recall the polytope $\mathcal{P}_{\lambda}=\operatorname{Conv}(W \cdot \lambda)$, which is the convex hull of $W \cdot \lambda$ in $X\left(T_{0}\right) \otimes \mathbb{Q}$, where $X\left(T_{0}\right)$ is the set of characters of $T_{0}$. The following corollary of Theorem 1.2 above describes the face lattice of $\mathcal{P}_{\lambda}$ in terms of the Weyl group $(W, S)$.

Corollary 1.3 Let $W$ be a Weyl group and let $r: W \rightarrow G L(V)$ be the usual reflection representation of $W$. Let $\mathcal{C} \subseteq V$ be the rational Weyl chamber and let 
$\lambda \in \mathcal{C}$. Assume that $J=\{s \in S \mid s(\lambda)=\lambda\}$. Then the set of orbits of $W$ acting on the face lattice $\mathcal{F}_{\lambda}$ of $\mathcal{P}_{\lambda}$ is in one-to-one correspondence with $\{I \subseteq S \mid$ no connected component of $I$ is contained entirely in $J\}$.

The subset $I \subseteq S$ corresponds to the unique face $F \in \mathcal{F}_{\lambda}$ with $I=\{s \in S \mid s(F)=$ $F$ and $s \mid F \neq i d\}$ whose relative interior $F^{0}$ has nonempty intersection with $\mathcal{C}$. See section 7.2 of [15] for a detailed discussion of the relationship between $\Lambda$ and the Weyl chamber.

Let $M$ be a $g$-irreducible monoid of type $J \subseteq S$ and let $\bar{T}$ be the closure in $M$ of a maximal torus $T$ of $G$. By part $b$ ) of Theorem 5.4 of [15], $\bar{T}$ is a normal variety. Define

$$
X(J)=[\bar{T} \backslash\{0\}] / \mathbb{C}^{*} .
$$

The terminology is justified since $X(J)$ depends only on $J$ and not on $M$ or $\lambda$. The set of distinct, normal $\mathrm{g}$-irreducible monoids associated with $X(J)$ can be identified with the set $\mathcal{C}^{J}=\left\{\lambda \in \mathcal{C} \mid C_{S}(\lambda)=J\right\}$. In the case $J=\emptyset, X(J)$ is the torus embedding studied in $[3,8,16]$.

\section{The augmented poset}

In this section we define the augmented poset $\left(W^{J}, \leq,\left\{v_{s}\right\}\right)$ associated with the subset $J$ of $S$. Recall that $W^{J} \subseteq W$ is the set of minimal length coset representatives of $W_{J}$ in $W$, and $\leq$ is the usual Bruhat ordering on $W_{J}$.

To achieve our objective we use some techniques from the theory of linear algebraic monoids. We use this theory to obtain some important results relating $W^{J}$ to a certain finite, partially ordered set $E_{1}$ of idempotents. That done, we obtain the desired "ascent/descent" structure on the poset $W^{J}$. See Proposition 2.17. Our construction has a fundamental relationship with the extremely important descent systems as discussed in Theorem 2.23 and Section 4. The reader who does not want to engage the monoids might be able to find his own proofs of Proposition 2.17 and Theorem 2.23 using his favorite techniques. See the table in Remark 2.24 for a handy translation between the monoid jargon and the Bruhat poset jargon. The theory of reductive monoids serves as an ideal method to help quantify the combinatorics of $W^{J}$ in geometric terms.

Let $M$ be a reductive, algebraic monoid with unit group $G$. Let $B \subseteq G$ be a Borel subgroup of $G$ and let $T \subseteq B$ be a maximal torus of $B$. As before $E(\bar{T})=\{e \in \bar{T}$ | $\left.e^{2}=e\right\}$ and $E_{1}(\bar{T})=\left\{e \in \bar{T} \mid e^{2}=e\right.$ and $\left.\operatorname{dim}(T e)=1\right\}$. As usual, $W$ is the Weyl group of $G$ relative to $T$. The next three technical results will allow us to find our way to the all-important Theorem 2.12.

Lemma 2.1 Let $e \in E_{1}(\bar{T})$. Then

$$
\overline{e B} \backslash\{0\}=\bigcup_{\tau \in X} e \tau B
$$

where $X=\left\{\tau \in W \mid e B \tau^{-1} e \neq 0\right\}$. 
Proof We first show that $\overline{e B} \backslash\{0\} \subseteq \bigcup_{\tau \in W} e \tau B$. To this end, first recall $e_{1} \in$ $E_{1}(\bar{T})$, the unique rank-one idempotent such that $e_{1} B=e_{1} B e_{1}$. Then $e_{1} G=$ $\bigsqcup_{w \in W} e_{1} B w B=\cup_{w \in W} e_{1} w B$, since $e_{1} B=e_{1} B e_{1}=\mathbb{C}^{*} e_{1}$. Thus, if $e=\gamma e_{1} \gamma^{-1} \in$ $E_{1}$, one sees that

$$
e G=\gamma e_{1} G=\bigcup_{w \in W} \gamma e_{1} B w B=\bigcup_{w \in W} \gamma e_{1} w B=\bigcup_{\tau \in W} e \tau B .
$$

Hence $\overline{e B} \backslash\{0\} \subseteq e G \subseteq \bigcup_{\tau \in W} e \tau B$.

Thus it suffices to show that $X=\{\tau \in W \mid e \tau \in \overline{e B}\}$. Suppose then, that $e \tau \in$ $\overline{e B}$. Then $0 \neq e \tau \tau^{-1} e \tau \in \overline{e B} \tau^{-1} e \tau$. Thus $e B \tau^{-1} e \tau \neq 0$. Conversely, suppose that $e B \tau^{-1} e \tau \neq 0$. Then there exists $b \in B$ such that $0 \neq x=e b \tau^{-1} e \tau$. Then $0 \neq x=$ $e x=x \tau^{-1} e \tau \in e B \tau^{-1} e \tau$. Thus $e \tau \in \mathbb{C}^{*} e \tau \subseteq e M \tau^{-1} e \tau=e B \tau^{-1} e \tau \subseteq \overline{e B}$ since $B \tau^{-1} e \tau \subseteq \bar{B}$.

Corollary 2.2 Let $e \in E_{1}(\bar{T})$ and let $f \in E(\bar{T})$. Then

$$
\overline{e B} f=\{0\} \cup\left(\bigcup_{\tau \in X} e \tau B f\right) .
$$

Proposition 2.3 The following are equivalent.

1. $e f=e$, and for all $\tau \in X$ with $\tau^{-1} e \tau \neq e$, e $\tau B f=0$.

2. $e B f=e B e$.

Proof Assume 1. Then, by Corollary 2.2,

$$
\overline{e B} f=\{0\} \cup\left(\bigcup_{\tau \in X} e \tau B f\right) .
$$

But, by assumption, $e \tau B f=0$ whenever $\tau^{-1} e \tau \neq e$. Hence $\overline{e B} f=\{0\} \cup$ $\left(\bigcup_{\tau \in Z} e \tau B f\right)$, where $Z=\left\{\tau \in X \mid \tau^{-1} e \tau=e\right\}$. However, if $\tau^{-1} e \tau=e$ then $e \tau=e$. Thus $\overline{e B} f=\{0\} \cup e B f=\{0\} \cup e f B f$, and this a closed subset of $M$. Using part (ii) of Corollary 7.2 of [11], we get ef $B f=e C_{B}(f)$. Thus $\overline{e B} f=e C_{B}(f) \cup\{0\}$, and hence $\overline{e B} f$ is the union of two right $C_{B}(f)$-orbits, $e C_{B}(f)$ and $\{0\}$. By part (i) of Theorem 6.16 of [11], $C_{B}(e)$ is a connected group. But it is also a solvable group. Thus, by Theorem 3.1 of [9], $\operatorname{dim}(e B f)=1$ since there exists $h \in \mathbb{C}[\overline{e B} f]$ such that $\{0\}=h^{-1}(0)$. Since $e B e \subseteq e B f$, it follows that $e B e=e B f$.

Conversely, assume 2. Thus $\overline{e B} f=\overline{e B e}=\{0\} \cup e B e$. But from Lemma 2.1 $\overline{e B} f=\{0\} \cup\left(\bigcup_{\tau \in X} e \tau B f\right)$. Assume that $e \tau B f \neq 0$. Then we have

$$
\emptyset \neq e \tau B f \backslash\{0\} \subseteq \overline{e B e} \backslash\{0\}=e B e=\mathbb{C}^{*} e .
$$

Thus,

$$
e \in e \tau B f \subseteq e \tau B f B \subseteq \overline{e \tau B}
$$

since $B f B \subseteq \bar{B}$. But $e \tau B \subseteq \overline{e B}$ and thus $e \tau B=e B$. Hence $e \tau=e$ and finally $\tau^{-1} e \tau=e$. 
Definition 2.4 Let $e, e^{\prime} \in E_{1}(\bar{T})$. We say that $e<e^{\prime}$ if $e B e^{\prime} \neq 0$ and $e \neq e^{\prime}$.

We shall see in Proposition 2.10 that $e<e^{\prime}$ if and only if $\overline{B e G} \subsetneq \overline{B e^{\prime} G}$. Then, in Theorem 2.12, we relate this to the Bruhat ordering on $W^{J}$, where $W_{J}$ is the centralizer in $W$ of $e_{1}$.

Theorem 2.5 Let $e \in E_{1}$ and let $f \in E$. The following are equivalent.

1. $e B f=e B e$.

2. (a) $e f=e$.

(b) If $e<e^{\prime}$ then $e^{\prime} B f=0$.

3. (a) $e f=e$.

(b) If $e<e^{\prime}$ then $e^{\prime} f=0$.

Proof The equivalence of 1 and 2 is a reformulation of Proposition 2.3, taking into account Definition 2.4. That 2 implies 3 is obvious. So we assume 3 and then deduce 1 . By Lemma 2.1

$$
\overline{e B} \backslash\{0\}=\bigcup_{\tau \in X} e \tau B
$$

where $X=\left\{\tau \in W \mid e B \tau^{-1} e \neq 0\right\}$. Now $e f=e$ so that $e B f=e f B f$. Thus $\overline{e B} f=$ $e \overline{f B f}=e \bar{C} f$, where $C=C_{B}(f)$. (Again using part(ii) of Corollary 7.2 of [11].) Thus, again by Proposition 2.3,

$$
e \bar{C} f \backslash\{0\}=\bigcup_{\gamma \in Y} e \gamma C f
$$

where $Y=\left\{\gamma \in W \mid e C \gamma^{-1} e \gamma \neq 0\right\}$. But if $e C e^{\prime} \neq 0$ then $e B e^{\prime} \neq 0$ and then, by assumption, $e^{\prime} f=0$ as long as $e^{\prime} \neq e$. Hence $e \gamma f=0$ if $\gamma^{-1} e \gamma \neq e$, and thus $e \gamma C f=e \gamma f C=0$ for $\gamma \in Y$. Thus $e B f=\{0\} \cup e C f$, which (as in the proof of Proposition 2.3) is one-dimensional. Thus $e B f=e B e$.

Notice how Theorem 2.5 allows us to describe the relationship of $B$ and $E$ in terms of the ordering $<$ on $E_{1}$.

Definition 2.6 Let $e \in E_{1}$. Define

$$
\mathcal{C}_{e}=\left\{f \in E(\bar{T}) \mid f e=e \text { and } f e^{\prime}=0 \text { for all } e^{\prime}>e\right\}
$$

From Theorem 2.5

$$
E(\bar{T}) \backslash\{0\}=\bigsqcup_{e \in E_{1}} \mathcal{C}_{e} .
$$

The reader is encouraged to think of $\mathcal{C}_{e} \subseteq E(\bar{T}) \backslash\{0\}$ as the combinatorial analogue of a $B B$-cell [1]. 
We recall now the Gauss-Jordan elements of $M$. First let $\mathcal{R}=\{x \in M \mid T x=$ $x T\} / T$. By the results of [13], $\mathcal{R}$ is a finite inverse monoid. Furthermore, there is a disjoint union decomposition

$$
M=\bigsqcup_{r \in \mathcal{R}} B r B .
$$

This monoid Bruhat decomposition is discussed in detail in Chapter 8 of [15]. It results in a perfect analogue, for reductive monoids, of the much-studied Bruhat decomposition of algebraic groups.

Definition 2.7 The set of Gauss-Jordan elements of $\mathcal{R}$ is defined to be

$$
G J=\{r \in \mathcal{R} \mid r B \subseteq B r\} .
$$

The following crucial properties of $G J$ are discussed in [13].

1. $G J \cdot W=\mathcal{R}$.

2. For each $x \in \mathcal{R}, G J \cap x W$ is a singleton.

3. $G J$ is a submonoid of $\mathcal{R}$.

4. $M=\bigsqcup_{r \in G J} B r G$.

The reader should think of the set of Gauss-Jordan elements of $\mathcal{R}$ as providing a combinatorial structure to the (generalized) Gauss-Jordan column-reduction algorithm. If $M$ is the reductive monoid of $n \times n$ matrices then one can check that, (relative to $T$ and $B$ the diagonal and upper-triangular matrices, respectively) $G J$ can be identified with the set of $0-1$ matrices, in reduced column echelon form, with at most one non zero entry in each row and column. See Section 8.3 of [15] for a detailed discussion of Gauss-Jordan elements for reductive monoids.

Proposition 2.8 The following are equivalent for $r, s \in G J$.

1. $B r G \subseteq \overline{B s G}$.

2. $B r \subseteq \overline{\overline{B s}}$.

Proof The case " 2 implies 1 " is clear. To prove " $l$ implies 2 " we shall use the fact that $B \backslash G$ is a complete variety. Since $s \in G J$ we have that $B s B=B s$. Thus $\overline{B s} B=\overline{B s}$. But then, by a result of Steinberg, $\overline{B s} G=\overline{B s G}$ since $B \backslash G$ is a complete variety. Thus the assumption of 1 is equivalent to saying that $B r G \subseteq \overline{B s} G$. Thus we can write $r=y g^{-1}$ where $y \in \overline{B s}$ and $g \in G$. Hence $r g \in \overline{B s}$. Thus $B r g B \subseteq \overline{B s}$. But $\operatorname{Brg} B=\operatorname{Br} B g B=B r B w B$ for some $w \in W$. But $1 \in \overline{B w B}$, and consequently $B r B \subseteq \overline{B r B w B}$. We conclude that $B r B \subseteq \overline{B S}$.

Recall that, for $J \subseteq S$,

$$
W^{J}=\left\{t \in W \mid t \text { has minimal length in } t W_{J}\right\} .
$$

Define also

$$
{ }^{J} W=\left\{t \in W \mid t \text { has minimal length in } W_{J} t\right\} .
$$

These will be required in the proof of the following theorem. 
Theorem 2.9 Let $r=v e_{1}, s=w e_{1}$ where $v, w \in W^{J}$. The following are equivalent.

1. $r \leq s$ (i.e. $B r B \subset \overline{B s B})$.

2. $w \leq v$ (i.e. $B w B \subset \overline{B v B})$.

Proof We apply Corollary 8.35 of [15]. But we notice first that, in that setup, $\Lambda$ is $\{e \in E(\bar{T}) \mid B e=e B e\}$ while in the present discussion, $\Lambda$ is $\{e \in E(\bar{T}) \mid e B=e B e\}$. To eliminate any potential confusion we shall first restate Corollary 8.35 using $\Lambda=$ $\{e \in E(\bar{T}) \mid e B=e B e\}$.

If $e, f \in \Lambda$ we write

$W_{I_{1}}=\{w \in W \mid w e=e w=e\}$ and $W_{I_{2}}=\{w \in W \mid w e=e w\}$,

and

$W_{J_{1}}=\{w \in W \mid w f=f w=f\}$ and $W_{J_{2}}=\{w \in W \mid w f=f w\}$.

Let $a, b \in \mathcal{R}$. Then $a=y^{-1} e x$ and $b=t^{-1} f u$ where $x \in^{I_{1}} W, y \in \in^{I_{2}} W, u \in^{J_{1}} W$ and $t \in{ }^{J_{1}} W$ (here $I_{1}, I_{2}, J_{1}, J_{2} \subseteq S$ ). This is the normal form for the elements of $\mathcal{R}$ as in Definition 8.34 of [15]. Then (from Corollary 8.35 of [15]) the following are equivalent.

i) $a \leq b$.

ii) $e f=e$, and there exists $w \in W_{I_{1}} W_{J_{2}}$ such that $x \leq w u$ and $w t \leq y$.

In our situation $W_{I_{1}}=W_{I_{2}}=W_{J_{1}}=W_{J_{2}}, x=u=1$ and $e=f=e_{1}$. So condition ii) becomes

ii)' There exists $w \in W_{I_{1}}$ such that $1 \leq w$ and $w t \leq y$.

which is equivalent to

ii)" $t \leq y$

since $t \leq w t$ for all $w \in W_{I_{1}}$. Now observe that $t \leq y$ if and only if $t^{-1} \leq y^{-1}$, while $\left({ }^{I} W\right)^{-1}=W^{I}$. Thus the result follows with $v=y^{-1}$ and $w=t^{-1}$.

Notice that this might appear counterintuitive. Think of $e_{1}$ as "large as possible on the left" and that, multiplication by some $w$ on the left makes the result smaller "on the left". Thus, if $w$ is less than $v$, then $v e_{1}$ is less than $w e_{1}$.

Proposition 2.10 The following are equivalent for $e, f \in E_{1}$.

1. $e<e^{\prime}$ (in the ordering of Definition 2.4 on $E_{1}$.).

2. $B e G \subset \overline{B e^{\prime} G}$.

Proof If $B e G \subset \overline{B e^{\prime} G}$ we first observe that $e \in e \overline{B e G} \neq 0$. But $e \overline{B e G} \subset \overline{B e^{\prime} G}$, and thus $e B e^{\prime} G \neq 0$. Hence $e B e^{\prime} \neq 0$.

Conversely, if $e B e^{\prime} \neq 0$ then $e B e^{\prime} G \neq 0$, and thus $e \overline{B e^{\prime} G} \neq 0$. But $e M=e G \cup\{0\}$ since $e \in E_{1}$. Thus $e \in e \overline{B e^{\prime} G}=e M$. But $e \overline{B e^{\prime} G} \subset \overline{B e^{\prime} G}$ since $e B \subset \bar{B}$. Thus $e \in$ $\overline{B e^{\prime} G}$ and finally $B e G \subset \overline{B e^{\prime} G}$. 
Remark 2.11 Notice that $B e G=B r G$ for $r \in W e_{1} \cap e W=\{r\}$. (See Section 8.3 of [15].) Similarly for $e^{\prime}$ and $s \in W e_{1} \cap e^{\prime} W=\{s\}$. Thus an equivalent statement is " $B r G \subset \overline{B s G}$ " for these $r, s \in G J$.

The following theorem is the "bridge" between the monoid geometry and the Bruhat combinatorics.

Theorem 2.12 The following are equivalent for $v, w \in W^{J}$.

1. $e=v e_{1} v^{-1}<e^{\prime}=w e_{1} w^{-1}$ in $\left(E_{1},<\right)$.

2. $w<v$ in $\left(W^{J},<\right)$, the Bruhat ordering on $W^{J}$.

Proof By Proposition 2.10,e $e e^{\prime}$ if and only if $B e G \subset \overline{B e^{\prime} G}$. As in Remark 2.11, let $B e G=B r G$ and $B e^{\prime} G=B s G$ where $r, s \in G J$.

By Proposition 2.8, $B r G \subset \overline{B s G}$ if and only if $B r \subseteq \overline{B s}$. Then by Theorem 2.9, $B r \subseteq \overline{B s}$ if and only if $w<v$, where $r=e v=v e_{1}, s=e^{\prime} w=w e_{1}$ and $v, w \in W^{J}$.

For $e \in E_{1}(\bar{T})$ we let

$$
\Gamma(e)=\left\{g \in E_{2}(\bar{T}) \mid g e=e \text {, and } g e^{\prime}=0 \text { for all } e^{\prime}>e\right\} .
$$

Corollary 2.13 Let $g \in E_{2}(\bar{T})$. Suppose that $e, f \in E_{1}(\bar{T})$ and that $e \neq f$. Assume that $g e=e$ and $g f=f$. Then either $e>f$ or else $f>e$. In particular

$$
\Gamma(e)=\left\{g \in E_{2}(\bar{T}) \mid g e=e, \text { and } g e^{\prime}=e^{\prime} \text { for some } e^{\prime}<e\right\}
$$

Proof Suppose that $e \ngtr f$. Recall Definition 2.6. Then $g \in \mathcal{C}_{f}$, since we have that $g e^{\prime}=0$ for any $e^{\prime}>f$. In particular, $g \notin \mathcal{C}_{e}$. Thus there exists $e^{\prime}>e$ such that $g e^{\prime}=$ $e^{\prime}$. But then $e^{\prime}=f$ since $g \in E_{2}$. Thus $f>e$.

Remark 2.14 If we think of $\leq$ as a relation on $E_{1}$ then Corollary 2.13 says that we can regard $E_{2}$ as a subrelation of $\leq$. Notice, in particular, that

$$
E_{2}=\bigsqcup_{e \in E_{1}} \Gamma(e) .
$$

In general we can identify $E_{1}$ and $E_{2}$ with the vertices and edges, respectively, of a certain polytope. See Remark 2.24 for a detailed explanation of how this works in the case of a g-irreducible monoid.

We now return to the case of a g-irreducible monoid. This is the case that is relevant to the discussion of descent systems. Recall that, in the general case, $\Lambda_{2}=$ $\left\{e \in E_{2} \mid e B=e B e\right\}$. But if $M$ is $g$-irreducible, it follows from part 2 of Theorem 1.2 
that, there is a canonical bijection

$$
\Lambda_{2} \cong S \backslash J
$$

This bijection is defined by

$$
s \rightsquigarrow g_{s},
$$

where $g_{s} \in \Lambda_{2}$ is the unique idempotent such that

1. $s g_{s}=g_{s} s \neq g_{s}$.

2. $g_{s} B \subseteq B g_{s}$.

See Theorem 4.13 of [12] for the detailed proof.

Since each $g \in \Gamma(e)$ is conjugate to one and only one $g_{s} \in \Lambda_{2}$ we can write

$$
\Gamma(e)=\bigsqcup_{s \in S \backslash J} \Gamma_{S}(e),
$$

where

$$
\Gamma_{s}(e)=\left\{g \in \Gamma(e) \mid g=v g_{s} v^{-1} \text { for some } v \in W\right\} .
$$

We now translate what we have learned from the monoids into results about Bruhat posets. Theorem 2.12 is the main result here that makes this possible. The following definition is the key ingredient that unifies our discussion.

Definition 2.15 Let $(W, S)$ be a Weyl group and let $J \subseteq S$ be a proper subset. Define

$$
S^{J}=\left(W_{J}(S \backslash J) W_{J}\right) \cap W^{J} .
$$

We refer to $\left(W^{J}, S^{J}\right)$ as the descent system associated with $J \subseteq S$.

Proposition 2.16 There is a canonical identification $S^{J} \cong\left\{g \in E_{2} \mid g e_{1}=e_{1}\right\}$.

Proof We first define

$$
\varphi: W_{J}(S \backslash J) W_{J} \rightarrow E_{1}
$$

by $\varphi(w)=w e_{1} w^{-1}$. Then $\varphi(w)=\varphi(v)$ if and only if $w W_{J}=v W_{J}$. Hence $\varphi$ induces an injection $\varphi: S^{J} \rightarrow E_{1}$. We now identify the image of $\varphi$. Let

$$
N\left(e_{1}\right)=\left\{e \in E_{1} \mid g e=e \neq e_{1} \text { and } g e_{1}=e_{1} \text { for some } g \in E_{2}\right\}
$$

and let $e \in E_{1}\left(e_{1}\right)$. Then there exists a unique $g \in E_{2}$ such that $g e=e$ and $g e_{1}=e_{1}$. By Proposition 6.27 of [11] and Theorem 4.13 of [12] there exists $u \in W_{J}$ such that

$$
g=u g_{s} u^{-1}
$$

for some unique $s \in S \backslash J$. But then $u s e_{1} s u^{-1}=e$, since $g f=f$ for exactly two rank-one idempotents $f$. It follows that

$$
\operatorname{image}(\varphi)=N\left(e_{1}\right) .
$$


The sought-after identification, $\theta: S^{J} \cong\left\{g \in E_{2} \mid g e_{1}=e_{1}\right\}$, is defined by

$$
\theta(w)=\left[e_{1}, \varphi(w)\right]
$$

where, by definition, $\left[e_{1}, \varphi(w)\right]$ is the unique rank-two idempotent $g$ such that $g e_{1}=$ $e_{1}$ and $g \varphi(w)=\varphi(w)$.

Proposition 2.17 Let $u, v \in W^{J}$ be such that $u^{-1} v \in S^{J} W_{J}$. In particular, $u \neq v$. Then either $u<v$ or $v<u$ in the Bruhat order $<$ on $W^{J}$.

Proof If $u, v \in W^{J}$ with $v=u r c, r \in S^{J}, c \in W_{J}$, consider as in Proposition 2.16, $g_{r} \in E_{2}\left(e_{1}\right)$. Then let $g=u g_{r} u^{-1}$. Then $g$ is the unique rank-two idempotent such that $g u e_{1} u^{-1}=u e_{1} u^{-1}$ and $g v e_{1} v^{-1}=v e_{1} v^{-1}$.

Recall from Theorem 2.12 that, for $u, v \in W^{J}$

$$
u e_{1} u^{-1}>v e_{1} v^{-1} \text { if and only if } u<v .
$$

But from Corollary 2.13, for $g \in E_{2}$ with $g e_{i}=e_{i}, i=2,3$, either $e_{2}>e_{3}$ or else $e_{3}>e_{2}$. The conclusion follows.

We let

$$
S_{s}^{J}=W_{J} s W_{J} \cap W^{J}
$$

Remark 2.18 Notice that

$$
S^{J}=\bigsqcup_{s \in S \backslash J} S_{S}^{J} .
$$

Indeed, by the proof of Proposition 2.16, $\theta: S^{J} \cong\left\{g \in E_{2} \mid g e_{1}=e_{1}\right\}$. Under this correspondence $S_{S}^{J}$ corresponds to $\left\{g \in E_{2} \mid g e_{1}=e_{1}\right.$ and $g=w g_{s} w^{-1}$ for some $\left.w \in W_{J}\right\}$.

Definition 2.19 Let $w \in W^{J}$. Define

1. $D_{s}^{J}(w)=\left\{r \in S_{s}^{J} \mid w r c<w\right.$ for some $\left.c \in W_{J}\right\}$, and

2. $A_{s}^{J}(w)=\left\{r \in S_{s}^{J} \mid w<w r\right\}$.

We refer to $D^{J}(w)=\sqcup_{s \in S \backslash J} D_{s}^{J}(w)$ as the descent set of $w$ relative to $J$, and $A^{J}(w)=\sqcup_{s \in S \backslash J} A_{s}^{J}(w)$ as the ascent set of $w$ relative to $J$.

By Proposition 2.17, for any $w \in W^{J}, S^{J}=D^{J}(w) \sqcup A^{J}(w)$.

Remark 2.20 Notice that $w r c<w$ for some $c \in W_{J}$ if and only if $(w r)_{0}<w$, where $(w r)_{0} \in w r W_{J}$ is the element of minimal length in $w r W_{J}$. See Example 4.3 for a revealing illustration of the fact that $S^{J}=D^{J}(w) \sqcup A^{J}(w)$. 
Definition 2.21 For each $v \in W^{J}$ and each $s \in S \backslash J$ define $v_{s}(v)=\left|A_{s}^{J}(v)\right|$. We refer to $\left(W^{J}, \leq,\left\{v_{s}\right\}\right)$ as the augmented poset of $J$. For convenience we let

$$
v(v)=\sum_{s \in S \backslash J} v_{S}(v) .
$$

Example 2.22 Let $(W, S)$ be the Weyl group of type $A_{3}$, so that $W=S_{4}$ and $S=$ $\left\{s_{1}, s_{2}, s_{3}\right\}$. Let $J=\phi$ and write $v_{i}$ for $v_{s_{i}}$. To keep track of all the numbers $\left\{v_{i}(w) \mid\right.$ $w \in W\}$ define

$$
H\left(t_{1}, t_{2}, t_{3}\right)=\sum_{w \in W} t_{1}^{\nu_{1}(w)} t_{2}^{\nu_{2}(w)} t_{3}^{\nu_{3}(w)}
$$

A straightforward calculation yields

$$
H\left(t_{1}, t_{2}, t_{3}\right)=1+\left(3 t_{1}+5 t_{2}+3 t_{3}\right)+\left(3 t_{2} t_{3}+5 t_{1} t_{3}+3 t_{1} t_{2}\right)+t_{1} t_{2} t_{3} .
$$

See Examples 4.3 and 4.4 below for a better illustration of how it works if $J \neq \emptyset$.

Theorem 2.23 Let $J \subset S$ be any proper subset. For $e=u e_{1} u^{-1}, u \in W$, we write $e=e_{u}$.

1. $E_{2} \cong\left\{(u, v) \in W^{J} \times W^{J} \mid u<v\right.$ and $\left.u^{-1} v \in S^{J} W_{J}\right\}$.

2. Let $u \in W^{J}$ and $e_{u}=u e u^{-1} \in E_{1}$. Then $E_{2}\left(e_{u}\right) \cong\left\{v \in W^{J} \mid u^{-1} v \in S^{J} W_{J}\right\}$.

3. Let $u \in W^{J}$ and $e_{u}=u e u^{-1} \in E_{1}$. Then $\Gamma\left(e_{u}\right) \cong\left\{v \in W^{J} \mid u<v\right.$ and $\left.u^{-1} v \in S^{J} W_{J}\right\} \cong A^{J}(u)$.

4. Let $u \in W^{J}$ and $e_{u}=u e u^{-1} \in E_{1}$. Then $\Gamma_{s}\left(e_{u}\right) \cong\left\{v \in W^{J} \mid u<v\right.$ and $\left.u^{-1} v \in S_{s}^{J} W_{J}\right\} \cong A_{s}^{J}(u)$.

5. If $w \in W^{J}$ and $s \in S \backslash J$ then $v_{s}(u)=\left|\Gamma_{s}\left(e_{u}\right)\right|$.

Proof This follows from Proposition 2.16 and Proposition 2.17.

Remark 2.24 The following table provides the reader with a summary-translation between the monoid jargon and the Bruhat poset jargon. Let $M$ be a $g$-irreducible monoid of type $J$, and let $\bar{T}$ be the closure in $M$ of a maximal torus. Let $E=E(\bar{T})$ be the set of idempotents of $\bar{T}$ and let $E_{i}=\{f \in E \mid \operatorname{dim}(f T)=i\} \subset E$. As above, we let $e_{1} \in E_{1}=E_{1}(\bar{T})$ be the unique element such that $e_{1} B=e_{1} B e_{1}$. For $e, e^{\prime} \in E_{1}$ let $v, w \in W^{J}$ be the unique elements such that $e=v e_{1} v^{-1}$ and $e^{\prime}=w e_{1} w^{-1}$. We write $e=e_{v}$ and $e^{\prime}=e_{w}$. For $e, f \in E$ we write $e \sim f$ if there exists $w \in W$ such that $w e w^{-1}=f$. If $s \in S \backslash J$ let $g_{s} \in E_{2}$ be the unique idempotent such that $g_{s} s=s g_{s}$ and $g_{s} B=g_{s} B g_{s}$. Let $\Lambda^{\times}=\{I \subset S \mid$ no component of $I$ is contained in $J\}$ and for $I \in \Lambda^{\times}$let $I^{*}=I \cup\{t \in J \mid t s=s t$ for all $s \in I\}$. 


\begin{tabular}{|l|l|}
\hline Reductive Monoid Jargon & Bruhat Order Jargon \\
\hline$e_{1} \in \Lambda_{1}=\left\{e_{1}\right\}$ & $1 \in W^{J}$ \\
\hline$e=e_{v} \in E_{1}$ & The $v \in W^{J}$ with $e=v e_{1} v^{-1}$ \\
\hline$e_{v} \leq e_{w}$ in $E_{1}$, i.e. $e_{v} B e_{w} \neq 0$ & $w \leq v$ in $W^{J}$ \\
\hline & $(u, v) \in W^{J} \times W^{J}$ such that \\
$E_{2}=\{g \in E \mid \operatorname{dim}(g T)=2\}$ & $u<v$ and $u^{-1} v \in S^{J} W_{J}$ \\
\hline$\left\{g \in E_{2} \mid g B=g B g\right\}$ & $S \backslash J$ \\
\hline$\left\{g \in E_{2} \mid g e_{1}=e_{1}\right\}$ & $S^{J}=\left(W_{J}(S \backslash J) W_{J}\right) \cap W^{J}$ \\
\hline$\left\{g \in E_{2} \mid g e_{1}=e_{1}, g \sim g_{s}\right\}$ & $S_{s}^{J}=\left(W_{J} s W_{J}\right) \cap W^{J}$ \\
\hline$E_{2}\left(e_{w}\right)=\left\{g \in E_{2} \mid g e_{w}=e_{w}\right\}$ & $\left\{v \in W^{J} \mid w^{-1} v \in S^{J} W_{J}\right\}$ \\
\hline$\Gamma\left(e_{w}\right)=\left\{g \in E_{2}\left(e_{w}\right) \mid g e^{\prime}=e^{\prime}\right.$ & $A^{J}(w)=\left\{r \in S^{J} \mid w<w r\right\}$ \\
\hline \multicolumn{1}{|c}{ for some $\left.e^{\prime}<e_{w}\right\}$} & \\
\hline$\Gamma_{s}\left(e_{w}\right)=\Gamma\left(e_{w}\right) \cap\left\{g \in E_{2} \mid g \sim g_{s}\right\}$ & $A_{s}^{J}(w)=\left\{r \in S_{s}^{J} \mid w<w r\right\}$ \\
\hline$E(\bar{T}) \backslash\{0\}$ & $\left\{(w, I) \mid I \in \Lambda^{\times}, w<w s\right.$ if $\left.s \in I^{*}\right\}$ \\
\hline
\end{tabular}

The "picture" here is this. The subset $W^{J} \subseteq W$ is canonically identified with the set of vertices of the rational polytope $\mathcal{P}_{\lambda}$. On the other hand there is a canonical ordering on $E_{1}=E_{1}(\bar{T})$ coming from the associated reductive monoid. Evidently $\left(E_{1}, \leq\right)$ and $\left(W^{J}, \leq\right)$ are anti-isomorphic as posets. Furthermore the set of edges $E d g\left(\mathcal{P}_{\lambda}\right)$ of $\mathcal{P}_{\lambda}$ is canonically identified with $E_{2}=E_{2}(\bar{T})$. If $g(v, w)=g(w, v) \in \operatorname{Edg}\left(\mathcal{P}_{\lambda}\right)$ is the edge of $\mathcal{P}_{\lambda}$ joining the distinct vertices $v, w \in W^{J}$ then either $v<w$ or else $w<v$. Given $v \in W^{J}$, with edges $E d g(v)=\left\{g \in E_{2} \mid g=g(v, w)\right.$ for some $\left.w \in W^{J}\right\}$, the question of whether $v<w$ or $w<v$ is coded in the "descent system" $\left(W^{J}, S^{J}\right)$.

\section{Bruhat posets and simple polytopes}

Recall that if $\lambda \in \mathcal{C}$, then the rational polytope $\mathcal{P}_{\lambda}$ records the combinatorial properties of the orbit structure of $T$ on $\bar{T}$. In this section we characterize, in terms of $J \subseteq S$, the conditions under which $\mathcal{P}_{\lambda}$ is a simple polytope. A polytope $\mathcal{P}$ is called simple if each vertex figure of $\mathcal{P}$ is a simplex, or equivalently, each vertex is the endpoint of exactly $m$ edges $\mathcal{P}$, where $m$ is the dimension of $\mathcal{P}$. An equivalent formulation is the following. Recall that $X(J)=[\bar{T} \backslash\{0\}] / \mathbb{C}^{*}$, where $\bar{T}$ is as above, with $J=\{s \in S \mid s(\lambda)=\lambda\}$. Then $\mathcal{P}_{\lambda}$ is a simple polytope if and only if $X(J)$ is rationally smooth.

Definition 3.1 We refer to $J$ as combinatorially smooth if $\mathcal{P}_{\lambda}$ is a simple polytope.

As usual we let $e_{1} \in E_{1}$ be the unique rank-one idempotent such that $e_{1} B=$ $e_{1} B e_{1}$. If $J \subseteq S$ we let $\pi_{0}(J)$ denote the set of connected components of $J$. To be more precise, let $s, t \in J$. Then $s$ and $t$ are in the same connected component of $J$ if there exist $s_{1}, \ldots, s_{k} \in J$ such that $s s_{1} \neq s_{1} s, s_{1} s_{2} \neq s_{2} s_{1}, \ldots, s_{k-1} s_{k} \neq s_{k} s_{k-1}$, and $s_{k} t \neq t s_{k}$. 
The following theorem indicates exactly how to detect the very interesting condition of Definition 3.1. We use, without mention, the natural correspondence between the face lattice of $\mathcal{P}_{\lambda}$ and the set of idempotents of $\bar{T}$. See Remark 2.24.

Theorem 3.2 Let $\lambda \in \mathcal{C}$. The following are equivalent.

1. $\mathcal{P}_{\lambda}$ is a simple polytope.

2. There are exactly $|S|$ edges of $\mathcal{P}_{\lambda}$ meeting at $\lambda$.

3. $J=\{s \in S \mid s(\lambda)=\lambda\}$ has the properties

(a) If $s \in S \backslash J$, and $J \nsubseteq C_{W}(s)$, then there is a unique $t \in J$ such that $s t \neq t s$. If $C \in \pi_{0}(J)$ is the unique connected component of $J$ with $t \in C$ then $C \backslash\{t\} \subseteq C$ is a setup of type $A_{l-1} \subseteq A_{l}$.

(b) For each $C \in \pi_{0}(J)$ there is a unique $s \in S \backslash J$ such that st $\neq t$ s for some $t \in C$.

Proof 1 and 2 are equivalent by standard results about polytopes.

Assume that 3 holds. We now deduce from this that 2 holds. This is equivalent to the statement $\left|\left\{f \in E_{2}(\bar{T}) \mid f e_{1}=e_{1}\right\}\right|=|S|$. Let $\Lambda_{2}=\{f \in \Lambda \mid \operatorname{dim}(f T)=2\}$, and recall that

$$
\Lambda_{2} \cong S \backslash J
$$

via the correspondence $f=f_{s}$ if $s f=f s \neq f$. See Theorem 4.16 of [12]. So we write

$$
\Lambda_{2}=\left\{f_{s} \mid s \in S \backslash J\right\} .
$$

Then from part (iii) of Proposition 6.27 of [11]

$$
\left\{f \in E_{2}(\bar{T}) \mid f e_{1}=e_{1}\right\}=\bigcup_{w \in W_{J}} w \Lambda_{2} w^{-1}=\bigsqcup_{s \in S \backslash J} C l_{W_{J}}\left(f_{S}\right)
$$

where $C l_{W_{J}}\left(f_{s}\right)$ is the $W_{J}$-conjugacy class of $f_{s}$. Let $s \in S \backslash J$.

Case 1: $s t=t s$ for all $t \in J$.

Then $f_{s} w=w f_{s}$ for all $w \in W_{J}$. In this case $C l_{W_{J}}\left(f_{s}\right)=\left\{f_{s}\right\}$.

Case 2: $t s \neq s t$ for some unique $t \in J$. Let $C$ be that unique connected component of $J$ with $t \in C$. Thus $C_{W_{J}}\left(f_{s}\right)=W_{J \backslash\{t\}}$ and, consequently, $C l_{W_{J}}\left(f_{s}\right) \cong W_{J} / W_{J \backslash\{t\}}$. But, by part (a) of the assumption,

$$
W_{J} / W_{J \backslash\{t\}} \cong W_{C} / W_{C \backslash\{t\}} \cong S_{m+1} / S_{m},
$$

where $|C|=m$ and $S_{m}$ is the symmetric group on $m$ letters. Thus

$$
\left|C l_{W_{J}}\left(f_{s}\right)\right|=\left|S_{m+1} / S_{m}\right|=\frac{(m+1) !}{m !}=m+1 .
$$

Since, by assumption, each $C$ occurs for exactly one $t \in S \backslash J$, we conclude that $\left|\left\{f \in E_{2}(\bar{T}) \mid f e_{1}=e_{1}\right\}\right|=\left[\sum_{C \in \pi_{0}(J)}(|C|+1)\right]+\mid\{s \in S \backslash J \mid s t=t s$ for all $t \in J\} \mid$. 
But $\sum_{C \in \pi_{0}(J)}(|C|+1)=|J|+\left|\pi_{0}(J)\right|$ while $\mid\{s \in S \backslash J \mid s t=t s$ for all $t \in J\} \mid=$ $|S \backslash J|-\left|\pi_{0}(J)\right|$. Thus, $\left|\left\{f \in E_{2}(\bar{T}) \mid f e_{1}=e_{1}\right\}\right|=|S|$.

Assume 2, and let $s \in S \backslash J$. As above,

$$
\left\{f \in E_{2}(\bar{T}) \mid f e_{1}=e_{1}\right\}=\bigsqcup_{s \in S \backslash J} C l_{W_{J}}\left(f_{s}\right) .
$$

If $s \in S \backslash J$ there are two cases.

Case 1: $s t=t s$ for all $t \in J$.

In this case $C l_{W_{J}}\left(f_{s}\right)=\left\{f_{s}\right\}$.

Case 2: $s t \neq t s$ for some $t \in J$.

For each such $t$ there is a unique $C \in \pi_{0}(J)$ such that $t \in C$. This is because the connected components of $J$ are disjoint.

One then checks that,

$$
C_{W_{C}}\left(f_{s}\right)=W_{C \backslash\{t\}},
$$

where $t \in C$ is the unique element such that $s t \neq t s$. ( $t$ is unique since $S$ is a tree).

But $W_{J}=\Pi_{C \in \pi_{0}(J)} W_{C}$ and $C_{W_{J}}\left(f_{s}\right)=\Pi_{C \in \pi_{0}(J)} C_{W_{C}}\left(f_{s}\right)$. Hence

$$
C l_{W_{J}}\left(f_{s}\right)=\Pi_{C \in V_{s}} C l_{W_{C}}\left(f_{s}\right)
$$

where $V_{s}=\left\{C \in \pi_{0}(J) \mid s t \neq t s\right.$ for some $\left.t \in C\right\}$.

Hence, for this $s \in S \backslash J$,

$$
\left|C l_{W_{J}}\left(f_{s}\right)\right|=\Pi_{C \in V_{s}}\left|C l_{W_{C}}\left(f_{s}\right)\right| .
$$

Thus

$$
\left|C l_{W_{J}}\left(f_{s}\right)\right|=\Pi_{C \in V_{s}}\left|W_{C} / W_{C \backslash\{t(s, C)\}}\right|,
$$

where $t(s, C)$ is the unique element of $C$ that fails to commute with $s \in S \backslash J$.

Combining Case 1 and Case 2, we obtain that

$$
\left|\left\{f \in E_{2}(\bar{T}) \mid f e_{1}=e_{1}\right\}\right|=|\operatorname{Int}(S \backslash J)|+\sum_{s \in B d(S \backslash J)} \Pi_{C \in V_{s}}\left|W_{C} / W_{C \backslash\{t(s, C)\}}\right|
$$

where $\operatorname{Int}(S \backslash J)=\{v \in S \backslash J \mid v t=t v$ for all $t \in J\}$ and $B d(S \backslash J)=\{v \in S \backslash J \mid$ $v t \neq t v$ for some $t \in J\}$. Notice that

$$
\pi_{0}(J)=\bigcup_{s \in B d(S \backslash J)} V_{s}
$$

since any connected component $C$ of $J$ contains at least one element that fails to commute with something in $S \backslash J$. Also it is a basic fact about Weyl groups that, if $C \subseteq S$ is connected and $t \in C$ then $\left|W_{C} / W_{C \backslash\{t\}}\right| \geq|C|+1$, with equality if and only if $C \backslash\{t\} \subseteq C$ is a setup of type $A_{l-1} \subseteq A_{l}$.

One checks that if the right-hand-side of this equation is equal to $|S|$ then all of the following must hold (since failure of any of them would make the RHS of (1) larger than $|S|)$. 
1. For each $s \in B d(S \backslash J)$, and for any $C \in V_{s}, C \backslash\{t(s, C)\} \subseteq C$ is a setup of type $A_{l-1} \subseteq A_{l}$.

2. For each $s \in B d(S \backslash J), V_{s}$ contains exactly one element.

3. For distinct elements $r, s \in S \backslash J, V_{s} \cap V_{r}=\emptyset$.

It then follows easily from this, that 3 holds.

In the next two examples one can use Equation 1 to calculate $\mid\left\{f \in E_{2}(\bar{T}) \mid f e_{1}=\right.$ $\left.e_{1}\right\} \mid$.

Example 3.3 Let $(W, S)$ be a Weyl group of type $A_{3}$, so that $S=\{r, s, t\}$ with $r s \neq s r$ and $s t \neq t s$.

(a) If $J=\{r, t\}$ then $\left|\left\{f \in E_{2}(\bar{T}) \mid f e_{1}=e_{1}\right\}\right|=4$. In this example $V_{s}=\{\{r\},\{t\}\}$, which violates condition 2. at the end of the proof of Theorem 3.2.

(b) If $J=\{s\}$ then $\left|\left\{f \in E_{2}(\bar{T}) \mid f e_{1}=e_{1}\right\}\right|=4$. In this example $V_{r}=V_{t}=\{\{s\}\}$ which violates condition 3. at the end of the proof of Theorem 3.2.

(c) If $J=\emptyset,\{r\}$, or $\{r, s\}$ then $\left|\left\{f \in E_{2}(\bar{T}) \mid f e_{1}=e_{1}\right\}\right|=3$. So these ones are combinatorially smooth.

Example 3.4 Let $(W, S)$ be a Weyl group of type $C_{3}$, so that $S=\{r, s, t\}$ with $r s \neq$ $s r$ and $s t \neq t s$, and $t$ corresponds to a short root. If $J=\{s, t\}$ then $\mid\left\{f \in E_{2}(\bar{T}) \mid\right.$ $\left.f e_{1}=e_{1}\right\} \mid=4$. In this example $\{t\} \subseteq\{s, t\}$ is a setup of type $A_{1} \subseteq C_{2}$ which violates condition 1. at the end of the proof of Theorem 3.2.

Notice in particular, if $(W, S)$ is an irreducible Weyl group and $J \subseteq S$ is a combinatorially smooth subset, then each connected component of $J$ contains exactly one end-node of $S$.

Corollary 3.5 For each irreducible Dynkin diagram we obtain the following calculation for $\{J \subseteq S \mid J$ is combinatorially smooth $\}$. For each type the list is grouped into the different cases depending on which of the end-nodes are elements of $J$.

1. $A_{1}$.

(a) $J=\phi$.

$A_{n}, n \geq 2$. Let $S=\left\{s_{1}, \ldots, s_{n}\right\}$.

(a) $J=\phi$.

(b) $J=\left\{s_{1}, \ldots, s_{i}\right\}, 1 \leq i<n$.

(c) $J=\left\{s_{j}, \ldots, s_{n}\right\}, 1<j \leq n$.

(d) $J=\left\{s_{1}, \ldots, s_{i}, s_{j}, \ldots, s_{n}\right\}, 1 \leq i, i \leq j-3$ and $j \leq n$.

2. $B_{2}$.

(a) $J=\phi$.

(b) $J=\left\{s_{1}\right\}$.

(c) $J=\left\{s_{2}\right\}$.

$B_{n}, n \geq 3$. Let $S=\left\{s_{1}, \ldots, s_{n}\right\}, \alpha_{n}$ short.

(a) $J=\phi$.

(b) $J=\left\{s_{1}, \ldots, s_{i}\right\}, 1 \leq i<n$.

(c) $J=\left\{s_{n}\right\}$. 
(d) $J=\left\{s_{1}, \ldots, s_{i}, s_{n}\right\}, 1 \leq i$ and $i \leq n-3$.

3. $C_{n}, n \geq 3$. Let $S=\left\{s_{1}, \ldots, s_{n}\right\}, \alpha_{n}$ long.
(a) $J=\phi$.
(b) $J=\left\{s_{1}, \ldots, s_{i}\right\}, 1 \leq i<n$.
(c) $J=\left\{s_{n}\right\}$.
(d) $J=\left\{s_{1}, \ldots, s_{i}, s_{n}\right\}, 1 \leq i$ and $i \leq n-3$.

4. $D_{n}, n \geq 4$. Let $S=\left\{s_{1}, \ldots, s_{n-2}, s_{n-1}, s_{n}\right\}$.
(a) $J=\phi$.
(b) $J=\left\{s_{1}, \ldots, s_{i}\right\}, i \leq n-3$.
(c) $J=\left\{s_{n-1}\right\}$.
(d) $J=\left\{s_{n}\right\}$.
(e) $J=\left\{s_{1}, \ldots, s_{i}, s_{n-1}\right\}, i \leq n-4$.
(f) $J=\left\{s_{1}, \ldots, s_{i}, s_{n}\right\}, i \leq n-4$.

5. $E_{6}$. Let $S=\left\{s_{1}, s_{2}, s_{3}, s_{4}, s_{5}, s_{6}\right\}$.
(a) $J=\phi$.
(b) $J=\left\{s_{1}\right\}$ or $\left\{s_{1}, s_{2}\right\}$.
(c) $J=\left\{s_{5}\right\}$ or $\left\{s_{4}, s_{5}\right\}$.
(d) $J=\left\{s_{6}\right\}$.
(e) $J=\left\{s_{1}, s_{5}\right\},\left\{s_{1}, s_{2}, s_{5}\right\}$ or $\left\{s_{1}, s_{4}, s_{5}\right\}$.
(f) $J=\left\{s_{1}, s_{6}\right\}$.
(g) $J=\left\{s_{5}, s_{6}\right\}$
(h) $J=\left\{s_{1}, s_{5}, s_{6}\right\}$.

6. $E_{7}$. Let $S=\left\{s_{1}, s_{2}, s_{3}, s_{4}, s_{5}, s_{6}, s_{7}\right\}$.
(a) $J=\phi$.
(b) $J=\left\{s_{1}\right\},\left\{s_{1}, s_{2}\right\}$ or $\left\{s_{1}, s_{2}, s_{3}\right\}$.
(c) $J=\left\{s_{6}\right\}$ or $\left\{s_{5}, s_{6}\right\}$.
(d) $J=\left\{s_{7}\right\}$.
(e) $J=\left\{s_{1}, s_{6}\right\},\left\{s_{1}, s_{2}, s_{6}\right\},\left\{s_{1}, s_{2}, s_{3}, s_{6}\right\},\left\{s_{1}, s_{5}, s_{6}\right\}$, or $\left\{s_{1}, s_{2}, s_{5}, s_{6}\right\}$.
(f) $J=\left\{s_{6}, s_{7}\right\}$.
(g) $J=\left\{s_{1}, s_{7}\right\}$ or $\left\{s_{1}, s_{2}, s_{7}\right\}$.
(h) $J=\left\{s_{1}, s_{6}, s_{7}\right\},\left\{s_{1}, s_{2}, s_{6}, s_{7}\right\}$.

7. $E_{8}$. Let $S=\left\{s_{1}, s_{2}, s_{3}, s_{4}, s_{5}, s_{6}, s_{7}, s_{8}\right\}$.
(a) $J=\phi$.
(b) $J=\left\{s_{1}\right\},\left\{s_{1}, s_{2}\right\},\left\{s_{1}, s_{2}, s_{3}\right\}$ or $\left\{s_{1}, s_{2}, s_{3}, s_{4}\right\}$.
(c) $J=\left\{s_{7}\right\}$ or $\left\{s_{6}, s_{7}\right\}$.
(d) $J=\left\{s_{8}\right\}$.
(e) $J=\left\{s_{1}, s_{7}\right\},\left\{s_{1}, s_{2}, s_{7}\right\},\left\{s_{1}, s_{2}, s_{3}, s_{7}\right\},\left\{s_{1}, s_{2}, s_{3}, s_{4}, s_{7}\right\}$, $\left\{s_{1}, s_{6}, s_{7}\right\},\left\{s_{1}, s_{2}, s_{6}, s_{7}\right\},\left\{s_{1}, s_{2}, s_{3}, s_{6}, s_{7}\right\}$ or $\left\{s_{1}, s_{2}, s_{5}, s_{6}\right\}$.
(f) $J=\left\{s_{7}, s_{8}\right\}$.
(g) $J=\left\{s_{1}, s_{8}\right\},\left\{s_{1}, s_{2}, s_{8}\right\}$ or $\left\{s_{1}, s_{2}, s_{3}, s_{8}\right\}$.
(h) $J=\left\{s_{1}, s_{7}, s_{8}\right\},\left\{s_{1}, s_{2}, s_{7}, s_{8}\right\}$.

8. $F_{4}$. Let $S=\left\{s_{1}, s_{2}, s_{3}, s_{4}\right\}$.
(a) $J=\phi$.
(b) $J=\left\{s_{1}\right\}$ or $\left\{s_{1}, s_{2}\right\}$.
(c) $J=\left\{s_{4}\right\}$ or $\left\{s_{3}, s_{4}\right\}$.
(d) $J=\left\{s_{1}, s_{4}\right\}$. 
9. $G_{2}$. Let $S=\left\{s_{1}, s_{2}\right\}$.
(a) $J=\phi$.
(b) $J=\left\{s_{1}\right\}$.
(c) $J=\left\{s_{2}\right\}$.

Proof This is an elementary calculation with Dynkin diagrams using Theorem 3.2. The numbering of the elements of $S$ is as follows. For types $A_{n}, B_{n}, C_{n}, F_{4}$, and $G_{2}$ it is the usual numbering. In these cases the end nodes are $s_{1}$ and $s_{n}$. For type $E_{6}$ the end nodes are $s_{1}, s_{5}$ and $s_{6}$ with $s_{3} s_{6} \neq s_{6} s_{3}$. For type $E_{7}$ the end nodes are $s_{1}, s_{6}$ and $s_{7}$ with $s_{4} s_{7} \neq s_{7} s_{4}$. For type $E_{8}$ the end nodes are $s_{1}, s_{7}$ and $s_{8}$ with $s_{5} s_{8} \neq s_{8} s_{5}$. In each case of type $E_{n}$, the nodes corresponding to $s_{1}, s_{2}, \ldots, s_{n-1}$ determine the unique subdiagram of type $A_{n-1}$. For type $D_{n}$ the end nodes are $s_{1}, s_{n-1}$ and $s_{n}$. The two subdiagrams of $D_{n}$, of type $A_{n-1}$, correspond to the subsets $\left\{s_{1}, s_{2}, \ldots, s_{n-2}, s_{n-1}\right\}$ and $\left\{s_{1}, s_{2}, \ldots, s_{n-2}, s_{n}\right\}$ of $S$.

Remark 3.6 It is easy to check that $J \subseteq S$ is combinatorially smooth if and only if $X(J)$ is rationally smooth. Indeed, this follows directly from Corollary 2 on page 136 of [4].

\section{The descent system $\left(W^{J}, S^{J}\right)$}

Let $(W, S)$ be a finite Weyl group and let $w \in W$. It is widely appreciated $[2,3,17]$ that the descent set

$$
D(w)=\{s \in S \mid l(w s)<l(w)\}
$$

determines a very large and important chapter in the study of Coxeter groups. In this section we interpret the results of Sections 2 and 3 solely in the language of Coxeter groups applied to $W, W^{J}, J \subseteq S$ and the Bruhat ordering on $W^{J}$. Our main result here is the explicit identification of the subset $S^{J} \subseteq W^{J}$.

Recall, from Definition 2.15, that

$$
S^{J}=\left(W_{J}(S \backslash J) W_{J}\right) \cap W^{J} .
$$

We refer to $\left(W^{J}, S^{J}\right)$ as the descent system associated with $J \subseteq S$.

Proposition 4.1 Let $\left(W^{J}, S^{J}\right)$ be the descent system associated with $J \subseteq S$. The following are equivalent.

1. $J$ is combinatorially smooth.

2. $\left|S^{J}\right|=|S|$.

3. $X(J)$ is rationally smooth.

Proof The equivalence of 1 and 2 follows from Proposition 2.16 using part 2 of Theorem 3.2. The equivalence of 1 and 3 follows from Remark 3.6.

Assume that $J \subseteq S$ is combinatorially smooth. Recall that, for $s \in S \backslash J$,

$$
S_{s}^{J}=\left(W_{J} s W_{J}\right) \cap W^{J} .
$$


Recall now, that for $s \in S \backslash J$, there is a unique $g_{s} \in \Lambda_{2}$ such that $\{s\}=\left\{t \in S \mid t g_{s}=\right.$ $\left.g_{s} t \neq g_{s}\right\}$. Furthermore, $s \rightsquigarrow g_{s}$ determines a bijection between $S \backslash J$ and $\Lambda_{2}$. Each $g \in E_{2}(\bar{T})$ is conjugate to a unique $g_{s}, s \in S \backslash J$. See part 2 of Theorem 1.2.

Theorem 4.2 Assume that $J \subseteq S$ is combinatorially smooth. Then

1. $S^{J}=\bigsqcup_{s \in S \backslash J} S_{s}^{J}$.

2. Let $s \in S \backslash J$. In case $s t=t s$ for all $t \in J, S_{s}^{J}=\{s\}$. Otherwise, $S_{s}^{J}=$ $\left\{s, t_{1} s, t_{2} t_{1} s, \ldots, t_{m} \cdots t_{2} t_{1} s\right\}$ where $C=C_{s}=\left\{t_{1}, t_{2}, \ldots, t_{m}\right\}, s t_{1} \neq t_{1} s$ and $t_{i} t_{i+1} \neq t_{i+1} t_{i}$ for $i=1, \ldots, m-1$.

3. $S_{s}^{J} \cong\left\{g \in E_{2} \mid g e_{1}=e_{1}\right.$ and $c g c^{-1}=g_{s}$ for some $\left.c \in W_{J}\right\}$.

Proof Part 1 follows from Remark 2.18. Part 2 follows from well-known information about the standard inclusion of symmetric groups $S_{n} \subseteq S_{n+1}$. See Theorem 3.2 above. Part 3 follows from Remark 2.18. See also the proof of Proposition 2.16.

Example 4.3 Let

$$
W=<s_{1}, \ldots, s_{n}>
$$

be the Weyl group of type $A_{n}$ (so that $W \cong S_{n+1}$ ), and let

$$
J=\left\{s_{2}, \ldots, s_{n}\right\} \subseteq S=\left\{s_{1}, \ldots, s_{n}\right\} .
$$

Then $J \subseteq S$ is combinatorially smooth. One checks, using Theorem 4.2, that

$$
W^{J}=\left\{1, s_{1}, s_{2} s_{1}, s_{3} s_{2} s_{1}, \ldots, s_{n} s_{n-1} \cdots s_{2} s_{1}\right\} .
$$

Notice that

$$
1<s_{1}<s_{2} s_{1}<\cdots<s_{n} s_{n-1} \cdots s_{1} .
$$

In this very special example we obtain that $S^{J}=W^{J} \backslash\{1\}$. Furthermore,

$$
A^{J}(w)=A_{s_{1}}^{J}(w)
$$

for each $w \in W^{J}$, since $S \backslash J=\left\{s_{1}\right\}$. Finally we obtain, by calculation, that

$$
\begin{aligned}
& \left(s_{j} \cdots s_{1}\right)\left(s_{1}\right)=\left[s_{j} \cdots s_{2}\right], \\
& \left(s_{j} \cdots s_{1}\right)\left(s_{i} \cdots s_{1}\right)=\left(s_{i-1} \cdots s_{1}\right)\left[s_{j} \cdots s_{2}\right] \text { if } 1<i \leq j, \text { and } \\
& \left(s_{j} \cdots s_{1}\right)\left(s_{i} \cdots s_{1}\right)=\left(s_{i} \cdots s_{1}\right)\left[s_{j+1} \cdots s_{2}\right] \text { if } i>j \geq 1
\end{aligned}
$$

We conclude from this that

$$
A^{J}\left(s_{j} \cdots s_{1}\right)=\left\{s_{m} \cdots s_{1} \mid m>j\right\} .
$$

Let us write $a_{j}=s_{j} \cdots s_{1}$ if we think of $s_{j} \cdots s_{1} \in W^{J}$, and $r_{j}=s_{j} \cdots s_{1}$ if we think of $s_{j} \cdots s_{1} \in S^{J}$. Also, if $w \in W$, we write $w_{0}$ for the element of minimal length in $w W_{J}$. By the calculation above we obtain that

$$
\left(a_{j} r_{i}\right)_{0}=1<a_{j} \text { if } 1=i \leq j,
$$


$\left(a_{j} r_{i}\right)_{0}=a_{i-1}<a_{j}$ if $1<i \leq j$, and

$\left(a_{j} r_{i}\right)_{0}=a_{i}>a_{j}$ if $i>j$.

Example 4.4 Let

$$
W=<s_{1}, \ldots, s_{n}>
$$

be the Weyl group of type $A_{n}$ (so that $W \cong S_{n+1}$ ), and let

$$
J=\left\{s_{3}, \ldots, s_{n}\right\} \subseteq S .
$$

Notice that $J \subseteq S$ is combinatorially smooth.

If $w \in W^{J}$ then $w=a_{p}, w=b_{q}$, or else $w=a_{p} b_{q}$. Here $a_{p}=s_{p} \cdots s_{1}(1 \leq$ $p \leq n)$ and $b_{q}=s_{q} \cdots s_{2}(2 \leq q \leq n)$. If we adopt the useful convention $a_{0}=1$ and $b_{1}=1$, then we can write

$$
W^{J}=\left\{a_{p} b_{q} \mid 0 \leq p \leq n \text { and } 1 \leq q \leq n\right\}
$$

with uniqueness of decomposition. Let $w=a_{p} b_{q} \in W^{J}$. After some tedious calculation with braid relations and reflections, we obtain that

a) $A_{s_{1}}^{J}\left(a_{p} b_{q}\right)=\left\{s_{1}\right\}$ if $p<q$.

$A_{s_{1}}^{J}\left(a_{p} b_{q}\right)=\emptyset$ if $q \leq p$.

Thus $v_{s_{1}}\left(a_{p} b_{q}\right)=1$ if $p<q$ and $v_{s_{1}}\left(a_{p} b_{q}\right)=0$ if $q \leq p$.

b) $A_{s_{2}}^{J}\left(a_{p} b_{q}\right)=\left\{s_{m} \cdots s_{n} \mid m>q\right\}$ if $q<n$.

$A_{s_{2}}^{J}\left(a_{p} b_{q}\right)=\emptyset$ if $q=n$.

Thus $v_{s_{2}}\left(a_{p} b_{q}\right)=n-q$.

It is interesting to compute the two-parameter "Euler polynomial"

$$
H\left(t_{1}, t_{2}\right)=\sum_{w \in W^{J}} t_{1}^{\nu_{1}(w)} t_{2}^{\nu_{2}(w)}
$$

of the augmented poset $\left(W^{J}, \leq,\left\{v_{1}, v_{2}\right\}\right)$ (where we write $v_{i}$ for $v_{s_{i}}$ ). A simple calculation yields

$$
H\left(t_{1}, t_{2}\right)=\sum_{k=1}^{n}\left[k t_{1}+(n+1-k)\right] t_{2}^{n-k} .
$$

\section{References}

1. Bialynicki-Birula, A.: Some theorems on the actions of algebraic groups. Annals of Math. 98, 480497 (1973)

2. Bjorner, A., Brenti, F.: Combinatorics of Coxeter Groups. Graduate Texts in Mathematics, vol. 231. Springer, New York (2005)

3. Brenti, F.: $q$-Eulerian poynomials arising from Coxeter groups. European Journal of Combinatorics 15, 417-441 (1994)

4. Brion, M.: Rational smoothness and fixed points of torus actions. Transformation Groups 4, 127-156 (1999) 
5. Brown, K.: Semigroup and ring theoretical methods in probability. In: Representations of Finite Dimensional Algebras and Related Topics in Lie Theory and Geometry. Fields Inst. Commun., vol. 40, pp. 3-26. Amer. Math. Soc., Providence (2004)

6. Danilov, V.I.: The geometry of toric varieties. Russian Mathematical Surveys 33, 97-154 (1978)

7. DeConcini, C., Procesi, C.: Complete Symmetric Varieties. Springer Lecture Notes in Mathematics, vol. 131. Springer, Berlin (1983), pp. 1-44

8. Dolgachev, I., Lunts, V.: A character formula for the representation of a Weyl group on the cohomology of the associated toric variety. Journal of Algebra 168, 741-772 (1994)

9. Grosshans, F.D.: The variety of points which are not semi-stable. Illinois Journal of Math. 26, 138-148 (1982)

10. Humphreys, J.E.: Introduction to Lie Algebras and Representation Theory, 3rd edn. GTM, vol. 9. Springer, Berlin (1980)

11. Putcha, M.S.: Linear Algebraic Monoids. Cambridge University Press, Cambridge (1988)

12. Putcha, M.S., Renner, L.E.: The system of idempotents and lattice of $\mathcal{g}$-classes of reductive algebraic monoids. Journal of Algebra 116, 385-399 (1988)

13. Renner, L.E.: Analogue of the Bruhat decomposion for algebraic monoids. Journal of Algebra 101, 303-338 (1986)

14. Renner, L.E.: An explicit cell decomposition of the canonical compactification of an algebraic group. Can. Math. Bull. 46, 140-148 (2003)

15. Renner, L.E.: Linear Algebraic Monoids. Encyclopedia of Mathematical Sciences, vol. 134. Springer, Berlin (2005)

16. Stembridge, J.R.: Some permutation representations of Weyl groups associated with the cohomology of toric varieties. Advances in Mathematics 106, 244-301 (1994)

17. Solomon, L.: A Mackey formula in the group ring of a Coxeter group. Journal of Algebra 41, 255-264 (1976)

18. Solomon, L.: An introduction to reductive monoids. In: Fountain, J. (ed.) Semigroups, Formal Languages and Groups, pp. 295-352. Kluwer Academic, Dordrecht (1995)

19. Stanley, R.P.: Log-concave and unimodal sequences in algebra, combinatorics and geometry. Ann. N.Y. Acad. Sci. 576, 500-534 (1989) 